\title{
Looking into the cradle: new mid-IR observations of multiple proto-stars ${ }^{\star}$
}

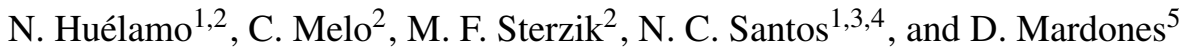 \\ ${ }^{1}$ Observatório Astronómico de Lisboa, Ed. Leste, Tapada da Ajuda, 1349-018 Lisboa, Portugal \\ e-mail: nhuelamo@aol.ul.pt \\ 2 European Southern Observatory, Alonso de Cordova 3107, Casilla 19001, Santiago 19, Chile \\ 3 Centro de Geofisica de Évora, Rua Romão Ramalho 59, 7002-554 Évora, Portugal \\ 4 Observatoire de Genève, 51 ch. des Maillettes, 1290 Sauverny, Switzerland \\ 5 Departamento de Astronomía, Universidad de Chile, Casilla Postal 36D, Santiago, Chile
}

Received 3 September 2006 / Accepted 22 November 2006

ABSTRACT

\begin{abstract}
Context. The multiplicity rate of the youngest stellar objects is directly linked to their formation process, and therefore represents one of the most critical parameters to constrain theories of star formation. Multiplicities of embedded protostars are, however, not well determined empirically.

Aims. The aim of this work is to study the multiplicity of three embedded proto-stellar systems, namely SSV 63, L1551 NE, and L1551-IRS5, through mid-IR imaging. All of them are suspected binary or multiple systems, but prone to ambiguities in the literature. Methods. We present high spatial resolution mid-infrared observations obtained with VISIR at the VLT.

Results. For the SSV 63 system, we report the discovery of a new Class I companion, approx 2".6 N of SSV63 E, which was never seen in previous near-IR and radio continuum studies. The companion to SSV $63 \mathrm{~W}$, which was detected at near-IR wavelengths, is also present in the mid-IR regime. In the case of L1551 NE, we have confirmed the presence of a companion at 0 .' $5 \mathrm{NW}$ from the central star, as previously reported by Reipurth et al. (2002, AJ, 124, 1045). Finally, we have not detected any companion to L1551IRS5. However, we find signs of extended emission around the target. In total, we have detected three binaries. The SEDs of the primaries are consistent with their Class I/flat-spectrum classification. For the secondaries, ancillary data are required to derive their evolutionary status.

Conclusions. The geometrical arrangement of sources in the SSV63 system strongly supports the view of hierarchical fragmentation of cloud cores.
\end{abstract}

Key words. stars: formation - stars: pre-main sequence - binaries: general

\section{Introduction}

Many young, low-mass, pre-main sequence stars are found in binaries or higher-order multiple systems. The observational evidence mainly stems from high angular resolution, near-infrared imaging surveys of significantly large samples of T Tauri stars (TTS, equivalent to Class II and III sources) probing different star-forming regions (for a summary see Mathieu \& Zinnecker 2000). Binary frequencies have been derived that are at least as high as for low-mass field dwarfs (Duquennoy \& Mayor 1991). Stellar multiplicities must therefore be established early in the stellar lifetime (Patience \& Duchêne 2001). Environmental specific differences between various star-forming associations and denser stellar clusters persist, and it is unclear if, e.g., different initial conditions in fragmenting cloud cores or dynamical processes in dense stellar clusters ultimately determine the multiplicity fractions (MF) and properties that are then observed in the T Tauri or later evolutionary phases (see a summary in Duchêne et al. 2006). It is therefore highly desirable to probe the MF for objects that are significantly younger than TTS, which have typical ages of $10^{6}$ years. The youngest stellar populations, Class 0 and Class I sources, are believed to have ages

* Based on observations collected at the La Silla-Paranal Observatory, ESO (Chile), with VISIR at Melipal under program ID 076.C-0518(A). between a few $10^{4}-10^{5}$ years, and are still deeply embedded in their star-forming cocoons. Not or only barely visible in the optical and near-infrared, advances in observing these sources are linked to the advent of sensitive, high angular-resolution techniques in the infrared and radio domain (e.g., lunar occultations, adaptive optics, interferometry).

In fact, the first systematic survey of embedded sources by Looney et al. (2000) revealed a surprisingly high MF, considering the low spatial resolution of the mm-continuum observations that only allowed to resolve extremely wide (>2000 AU) systems. In systematic VLA studies, Reipurth (2000) and Reipurth et al. (2002, 2004) probed a larger sample of mostly Class I sources down to sub-arcsec separations. High MF were confirmed, and the conjectured that strong outflows from embedded sources are related to periodical dynamical interactions within primordial higher-order multiple systems. Adaptiveoptics-assisted near-infrared imaging allowed Duchêne et al. (2004, 2006, in prep.) to discover new, faint, sub-arc companions to Class I protostars in different star-forming regions. In contrast to the apparent differences of the MF of TTS in Orionlike (dense cluster) and Taurus-like (associations) environments, Class I multiple systems have the same, high MF (approx. 52\% according to Duchêne et al. 2004) in all clouds. These observations strengthen the notion that multiplicity is universal and begins with birth. 
Table 1. VISIR observation Log.

\begin{tabular}{llllllll}
\hline \hline Target & Obs. date & Filter & $\begin{array}{c}\text { Exp. time } \\
{[\mathrm{s}]}\end{array}$ & Airmass ${ }^{1}$ & Calibrator & Airmass calib. & $\begin{array}{c}\text { Sensitivity }^{2} \\
{[\mathrm{mJy} / 10 \sigma / 1 \mathrm{~h}]}\end{array}$ \\
\hline SSV 63 & $2005-12-11$ & $P A H 1$ & 1615.0 & 1.3 & HD 39425, HD 99998 & $1.02,1.24$ & 4.8 \\
SSV 63 & $2006-03-14$ & SIV & 1965.6 & 1.3 & HD 41047, HD 37984 & $1.03,1.45$ & 9.5 \\
SSV 63 & $2005-12-12$ & PAH2 & 2710.7 & 1.2 & HD 41047, HD 29085 & $1.08,1.20$ & 8.5 \\
SSV 63 & $2006-03-02$ & $P A H 2$ & 1259.5 & 1.3 & HD 61935, HD 75691 & $1.00,1.04$ & 7.5 \\
\hline L1551 NE & $2006-02-12$ & PAH1 & 2697.0 & 1.6 & HD 26967, HD 75691 & $1.10,1.01$ & 6.2 \\
L1551 NE & $2006-03-05$ & SIV & 2683.0 & 1.7 & HD 25165, HD 41047 & $1.58,1.01$ & 9.8 \\
L1551 NE & $2006-03-04$ & $P A H 2$ & 2710.7 & 1.7 & HD 41047, HD 61935 & $1.05,1.01$ & 6.5 \\
\hline L1551-IRS5 & $2006-03-03$ & $P A H 1$ & 2339.3 & 1.8 & HD 61935 & 1.04 & 8.9 \\
L1551-IRS5 & $2006-02-11$ & SIV & 2683.2 & 1.7 & HD 26967 & 1.01 & 1.4 \\
L1551-IRS5 & $2006-03-17$ & SIV & 1786.2 & 2.0 & HD 41047 & 1.02 & 10.7 \\
L1551-IRS5 & $2005-12-26$ & $P A H 2$ & 1802.7 & 1.6 & HD 26967, HD 61935 & $1.06,1.04$ & 10.0 \\
L1551-IRS5 & $2006-03-02$ & $P A H 2$ & 2348.2 & 1.8 & HD 41047, HD 61935 & $1.01,1.04$ & 7.3 \\
\hline
\end{tabular}

Notes: ${ }^{1}$ average value during the exposure; ${ }^{2}$ derived from standard star observations.

However, the earliest, more embedded, stages of low-mass star formation are still poorly constrained by observations. Multi-wavelength data are required to constrain the spectral energy distribution, and therefore the evolutionary state. High spatial resolution and sensitivity is needed to probe small scales for binarity. Near-IR high resolution spectroscopy may also become a promising tool for detecting the closest, spectroscopic companions (Covey et al. 2006).

We have therefore started a program to systematically study the environment of protostars in the mid-infrared with VISIR at the VLT, allowing diffraction limited imaging down to 0.3 . In this paper we report the first results of our survey targeted on three protostars located in Taurus (L1551-IRS5 and L1551 NE) and Orion (SSV 63). All three targets are prominent IRAS sources, and have been reported to be suspected binary or multiple systems in the literature. We present the first highresolution MIR images of these protostars, and clarify the physical properties and multiplicity status of these sources.

\section{Observations and data analysis}

The data presented here were acquired with the VLT Imager and Spectrograph for mid-IR, VISIR, mounted on Melipal/UT3 (Lagage et al. 2004). We selected a pixel scale of $0.075^{\prime \prime} /$ pix, which provides a total FOV of $19 . ' 2 \times 19$ !' 2 . The observations were performed using standard chopping and nodding techniques to suppress the sky emission. We used chop throws of 7'.'0, 10.' 0 and 15.'0 for SSV63, L1551 NE, and L1551-IRS5, respectively. The three protostars were observed in three filters, namely, PAH1 $\left(\lambda_{\mathrm{o}} / \Delta \lambda=8.59 / 042 \mu \mathrm{m}\right)$, SIV $(10.49 / 0.16 \mu \mathrm{m})$, and PAH2 $(11.25 / 0.59 \mu \mathrm{m})$, which allow us to study the behavior of the silicate feature at $9.8 \mu \mathrm{m}$. The data were acquired with service mode queued observations. Some of the targets were observed more than once until the observational constraints were fulfilled. We have analyzed all available data and used redundant observations whenever possible. The observational details are summarized in Table 1.

The data have been reduced with a customary pipeline based on Interactive Data Language (IDL) routines. The final images are the result of shifting and combining the individual chopping cycles and removing those with bad image quality. The fluxes of SSV 63 and L1551-IRS5 have been measured performing aperture photometry (Table 2). We have derived count-rate-to-energy conversion factors from the spectrophotometric standard star observations. Note that both L1551-IRS5 and L1551 NE were observed at high airmass, while the standard stars were observed at zenith. Hence, the derived fluxes may be underestimated by up to $20 \%$ (PAH1), $8 \%$ (PAH2) and $2 \%$ (SIV) $)^{1}$.

Since L1551 NE is a close binary, we have performed PSFfitting and subtraction to derive the separation, position angle (PA), and flux ratio of the two sources in the SIV and PAH2 images. This ratio, together with the total flux, has been used to derive the emission from the individual components. In the PAH1 image, the secondary is faint and the PSF of the primary elongated, so PSF-fitting does not provide conclusive results. We have recomputed the binary parameters using a second and independent method: we have reflected the SIV and PAH2 images about the perpendicular axis to the line connecting the two stars, re-aligned them, and subtracted them from the original ones. In this way, the light from the primary is removed and we can estimate the position and flux of the companion. We obtain separations and PA consistent with those from PSF-subtraction methods. The flux ratios, $[b / a]$, derived with the two methods vary between $0.15-0.2$ for the SIV and $0.14-0.2$ for the PAH2. We have assumed an average value of 0.17 . The values are listed in Table 3.

\section{Results}

\section{1. $S S V 63$}

SSV 63 (Strom et al. 1976) is associated with the HH 24-26 active star-forming region in the L1630 Orion molecular complex. Narrow-band $2.12 \mu \mathrm{m}$ images by Zealey et al. (1992) resolve it in two sources SSV63E and SSV63W, separated by $\sim 8^{\prime \prime}(0.02 \mathrm{pc})$. Bontemps et al. (1995) confirm them in deep $3.6 \mathrm{~cm}$ radio continuum observations. $J H K$ observations showed that SSV 63 is accompanied by two additional nebulae NE-1 and NE-2 (Moneti \& Reipurth 1995) at a distance of approx. $14^{\prime \prime}$. Note that the separation of these two cloudlets is $\sim 7 \prime .5$, so that only SSV 63NE-1 is within the VISIR FOV. Davis et al. (1997) present higher resolution near-infrared narrow-band images. Sources $\mathrm{E}$ and $\mathrm{W}$ looked extended and the latter is resolved into two sources. Based on theoretical considerations of precession of collimated outflows, and their observational appearance, Terquem et al. (1999) conjecture that the SSV 63 system must be a hierarchical system of four or even five young stars. They postulate that component $\mathrm{W}$ is a 920 AU binary (based on

\footnotetext{
1 Based on a robust linear fit of the conversion factor as a function of airmass using relevant VISIR standard star observations.
} 


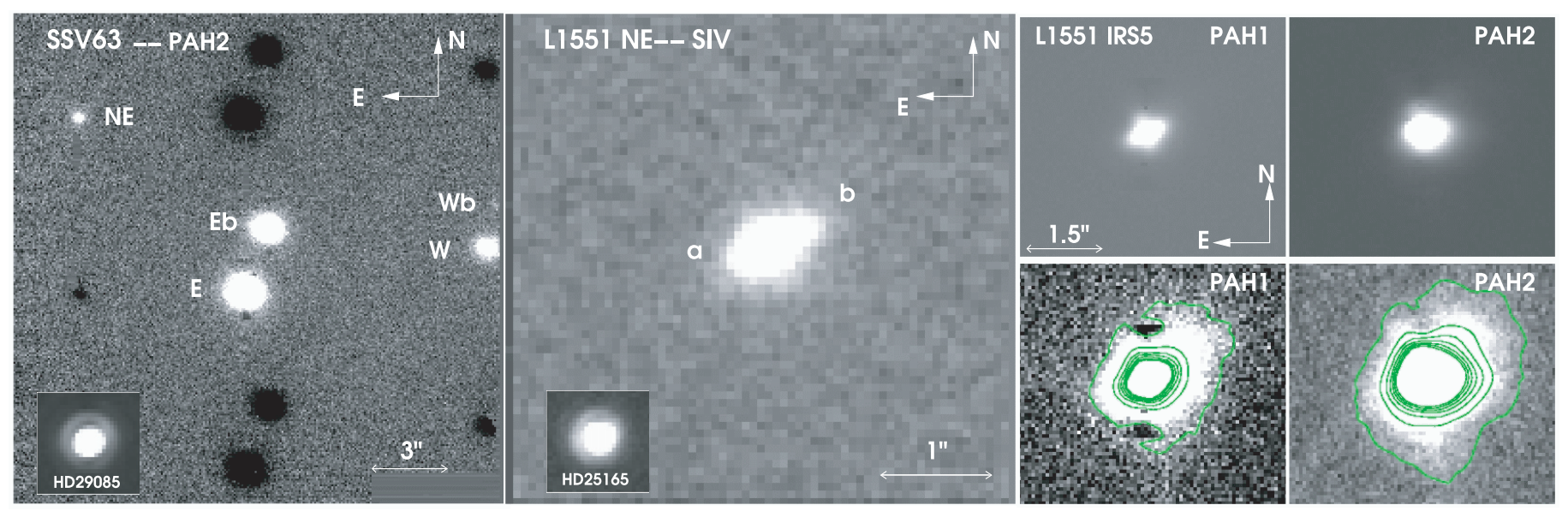

Fig. 1. Left and center: VISIR images of SSV63 and L1551NE. The field-of-view is $\sim 19^{\prime \prime} \times 19^{\prime \prime}$ and $\sim 5^{\prime \prime} \times 5^{\prime \prime}$, respectively. Images of standard stars observed at similar airmasses are displayed in the bottom left panels. Right: VISIR images of L1551 IRS5. The top and bottom panels are displayed with different cut levels to distinguish the PSF from the suspected extended emission around the target. The dark features in the PAH1 image (bottom) are electronic artifacts.

Davis et al. 1997 images), and component E actually a triple system, with projected separations of 975 AU and 350 AU. Further high resolution VLA observations of Reipurth et al. (2002) reveal only three sources within a square of $20^{\prime \prime}$ centered around SSV 63: SSV 63E, SSV 63W, and SSV 63NE.

Our own MIR VISIR images (Fig. 1, left) clarify the distribution of sources in that field. $2^{\prime \prime} 6$ north $\left(\mathrm{PA}=340.8 \pm 0.3^{\circ}\right)$ of our central pointing towards SSV 63E, we find a strong MIR source that we call Eb. From Table 3 we infer that source Eb is actually significantly bluer then $\mathrm{E}$, and apparently exhibits $\mathrm{Si}$ absorption, as its flux in the $S I V$ filter bandpass is decreased with respect the neighboring bands. Future MIR spectroscopy is required to compare potential differences in the mineralogy of both sources. Assuming a distance of 480 pc (like Terquem et al. 1999), the projected separation of both sources is $1248 \mathrm{AU}$, roughly compatible with the postulate by Terquem et al. (1999). However, we are unable to identify a third, closer, component.

Source $\mathrm{W}$ is clearly identified 9.'6 $\left(\mathrm{PA}=280.5 \pm 0.1^{\circ}\right)$ towards the west. In the $P A H$ filters we can identify another source, $\mathrm{Wb}$, lying just at the edge of our VISIR frame ( $\mathrm{Wb}$ is out of the field of view in the SIV image). This binary was already resolved in the near-IR by Davis et al. (1997). We measure a separation of $1^{\prime \prime} .7\left(\mathrm{PA}=351 \pm 1^{\circ}\right)$, corresponding to $816 \mathrm{AU}$, somewhat smaller, but roughly in accord with the 920 AU measured by Terquem et al. (1999). A fifth point-like source, SSV $63 \mathrm{NE}$, is located 9.' $5\left(\mathrm{PA}=46.2 \pm 0.2^{\circ}\right)$ northeast of our reference source, SSV 63E, in agreement with the position of a $3.6 \mathrm{~cm}$ radio source found by Reipurth et al. (2002). VISIR observations do not show additional companions to this MIR source.

We construct the spectral energy distributions (SEDs) for the five SSV 63 objects for which we were able to measure MIR photometry (Table 2). The near-IR ( $J H K)$ data have been adopted from Moneti \& Reipurth (1995). The slope of the SED between the near-IR and mid-IR has been used to study the evolutionary status of young stellar objects (e.g., Lada 1987). A least-square fit to the data from 2.2 to $11.3 \mu \mathrm{m}$ provides slopes of $\alpha=0.82$ and 0.25 for the $\mathrm{E}$ and $\mathrm{W}$ components, consistent with Class-I and flat-spectrum objects, respectively.

The detected secondaries (together with the NE source) do not have unambiguous near-infrared measurements, and thus do not allow us to clearly infer their evolutionary state. From the absence of clear detections and the sensitivity limits of
Table 2. Mid-IR photometry.

\begin{tabular}{llll}
\hline \hline Target & $\begin{array}{l}\text { PAH1 } \\
{[\mathrm{mJy}]}\end{array}$ & $\begin{array}{l}\text { SIV } \\
{[\mathrm{mJy}]}\end{array}$ & $\begin{array}{l}\text { PAH2 } \\
{[\mathrm{mJy}]}\end{array}$ \\
\hline SSV 63 E & $621.3 \pm 22.2$ & $761.9 \pm 30.9$ & $1011.2 \pm 150.0$ \\
SSV 63 Eb & $709.1 \pm 25.9$ & $357.4 \pm 17.0$ & $481.0 \pm 45.0$ \\
SSV 63 W & $114.0 \pm 10.0$ & $158.0 \pm 16.0$ & $170.5 \pm 20.0$ \\
SSV 63 Wb & $6.5 \pm 1.5$ & na & $9.6 \pm 2.0$ \\
SSV 63 NE & $60.6 \pm 10.0$ & $42.8 \pm 6.2$ & $49.1 \pm 6.5$ \\
L1551 NE a & $405 \pm 10^{1,2}$ & $239 \pm 15.0$ & $339 \pm 20^{1}$ \\
L1551 NE b & na & $42 \pm 10$ & $58 \pm 12^{1}$ \\
L1551-IRS5 & $3004 \pm 150^{1}$ & $3629 \pm 100^{1}$ & $5625 \pm 500^{1}$ \\
\hline
\end{tabular}

Notes: ${ }^{1}$ fluxes may be underestimated by up to $20 \%$ (PAH1), $8 \%$ (PAH2), and $2 \%$ (SIV), see text. ${ }^{2}$ Combined flux of components $a$ and $b$.

Table 3. Binary parameters.

\begin{tabular}{lllll}
\hline \hline Target & $\begin{array}{l}\text { Sep. } \\
{\left[^{\prime \prime}\right]}\end{array}$ & $\begin{array}{l}\text { PA } \\
\left({ }^{\circ}\right)\end{array}$ & {$[b / a]_{8.6}$} & {$[b / a]_{11.3}$} \\
\hline SSV 63 E-Eb & $2.60 \pm 0.02$ & $340.8 \pm 0.3$ & 1.14 & 0.47 \\
SSV 63 W-Wb & $1.70 \pm 0.05$ & $351 \pm 1$ & 0.06 & 0.06 \\
L1551 NEa-b & $0.45 \pm 0.05$ & $294 \pm 4$ & - & 0.17 \\
\hline
\end{tabular}

Moneti \& Reipurth (1995) and Davis et al. (1997), we believe that the near infrared counterparts are faint, and deeply embedded. They might be in a very early evolutionary state, or have a very low mass. Further sensitive and higher spatial resolution near infrared imaging is needed to characterize the SED of these sources.

\section{2. $L 1551 N E$}

L1551 NE was first suggested to be a binary source by Rodríguez et al. (1995) based on $3.5 \mathrm{~cm}$ low signal-to-noise observations. Later Moriarty-Schieven et al. (2000) confirmed the binary nature of L1551 NE. However, the position of the B-component was in clear contradiction to that claimed by Rodríguez et al. (1995). More recently Reipurth et al. (2002) carried out additional $3.6 \mathrm{~cm}$ high signal-to-noise VLA observations in which both components of L1551 NE are clearly seen. The system is a binary with a separation of $0 .^{\prime} 5$ at a PA of $297^{\circ}$, consistent with 
Rodríguez et al.'s (1995) result. In the VLA image, the SE component $(a)$ is brighter, whereas the NW component $(b)$ is $\sim 0.7$ fainter.

In Fig. 1 (center) we show our VISIR image in the SIV filter. Our mid-IR images confirm L1551 NE as a binary with a measured separation of $0 \prime^{\prime} 45 \pm 00^{\prime} 05$ and a PA of $294^{\circ} \pm 4$ in good agreement with those quoted by Reipurth et al. (2002). From our photometry we measured SIV and PAH2 flux ratios of $[b / a] \sim 0.17$, which are much smaller than the 0.7 found by Reipurth et al. (2002). This indicates that component $b$ may be heavily extincted, and even more embedded than $a$. No additional components are seen in the MIR images.

We sketch the SED of this system in the top panel of Fig. 3. The Infrared Space Observatory (ISO) spectrum of the system (retrieved from the public archive) is overplotted for wavelengths longward of $30 \mu \mathrm{m}$. Near-infrared photometry and the PAH1 photometry correspond to the whole system flux, while SIV and PAH2 have been separated for the two sources. From the shape of the SED there is little doubt that the system is in a very early evolutionary phase.

\section{3. $L 1551-I R S 5$}

L1551-IRS 5 is known to be a double system since Bieging \& Cohen (1985) detected it at centimeter radio continuum wavelengths. Both sources are surrounded by disks and power collimated jets (Rodríguez et al. 2003). Lim \& Takakuwa (2005) have presented high spatial resolution ( $\sim 30$ mas) observations obtained with the VLA at $7 \mathrm{~mm}$, which show that the system is a triple, with companions located at 0.326 S and 0.'079 SE from the primary, that is, $46 \mathrm{AU}$ and $11 \mathrm{AU}$ at the distance of Taurus ( $d=140$ pc, Kenyon \& Hartmann 1995). Covey et al. (2006) have not reported a significant radial velocity dispersion for this source.

The diffraction limit of VLT/VISIR at $8.6 \mu \mathrm{m}$ is $\sim 0.26$, which would allow us to detect the wider companion at $46 \mathrm{AU}$. Unfortunately, the VISIR observations were carried out at relatively high airmasses, and suffered from image quality degradation and unstable PSF. Moreover, all the observations but the PAH1 ones were seeing-limited. The FWHM of the target in the best PAH1 cycle is only 0!'36. The PSF is strongly asymmetric and the source is elongated in the SE-NW direction. The FWHM of the PAH2 images is 0.'39 in the best cycles, but the primary is slightly elongated in the WE direction. As shown in Fig. 1 (right), we have not detected any companions close to the source.

We have estimated the largest detectable difference of magnitude between the primary and a secondary, as a function of the projected separation. The limits of sensitivity have been computed from the average of the $3-\sigma$ noise measurements on the radial profile of the star. Figure 4 shows the results. The strong asymmetry of the PAH1 PSF translates into higher noise and worse sensitivities at smaller separations. The flux ratios derived at the separation of the secondary $(0.326)$ are 0.3 for the PAH1 and 0.16 for the SIV and PAH2 filters, which provide upper limits of $~ 900 \mathrm{mJy}$ (PAH1 and PAH2) and $580 \mathrm{mJy}$ (SIV).

On the other hand, the target seems to be surrounded by extended emission (Fig. 1, right bottom) which is present in the two PAH filters, but is not obvious in SIV. The lack of standard stars observed at similar airmasses prevents us from performing PSF-subtraction and drawing final conclusions about this faint, extended emission. Although we think it is real, additional observations are required to confirm it.

The SED of L1551-IRS5, including the ISO spectrum, our MIR measurements and the $J H K$ fluxes, is displayed in the

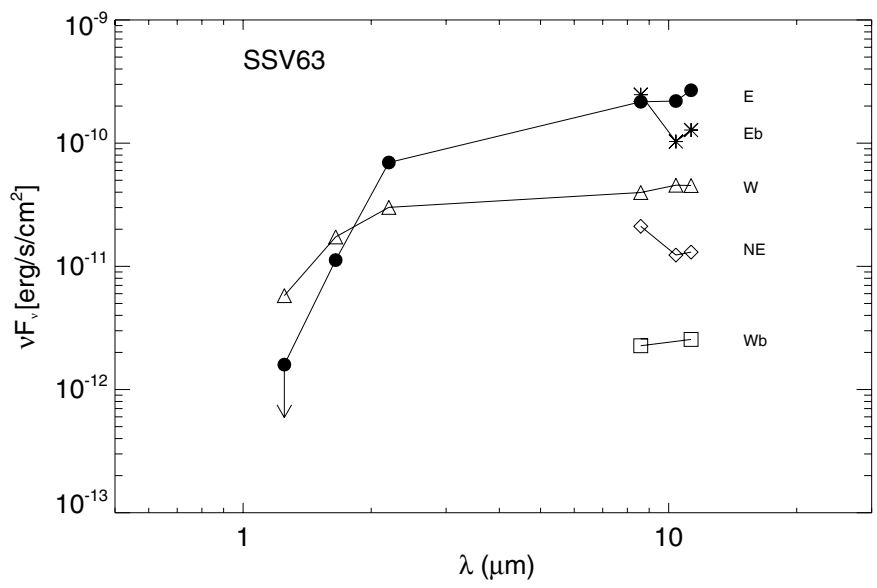

Fig. 2. SEDS of the protostars in the SSV63 field in Orion. The near-IR data have been adopted from 2MASS.
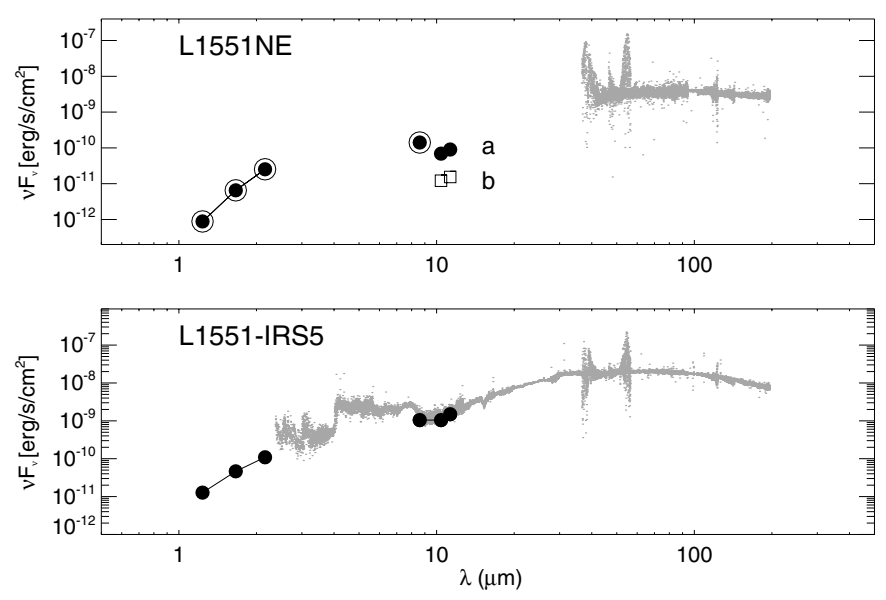

Fig. 3. SEDS of the two protostars L1551NE and L1551-IRS5 in Taurus. ISO spectra are overplotted. In the case of L1551NE, the big circles represent the flux measured for the whole binary system, $a+b$, in the near-IR and PAH1.
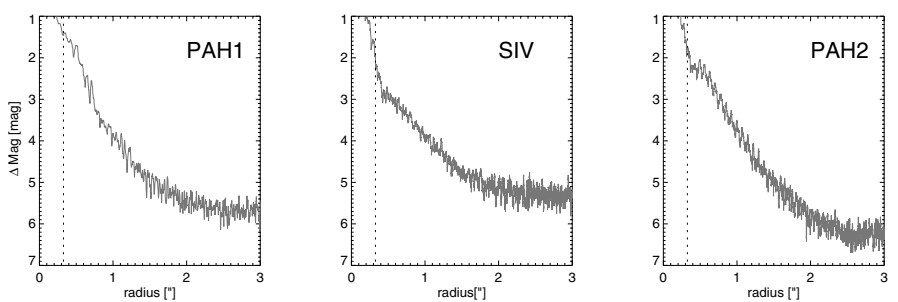

Fig. 4. Detection limits as a function of the separation from L1551IRS5. We measure $\Delta \mathrm{Mag}[\mathrm{mag}]$ of 1.4 for the PAH1 and $\sim 2.0$ for the SIV and PAH2 at a separation of 0 ' 326.

bottom panel of Fig. 3. If this system is a binary or triple system, the components must indeed be closer than our resolution limit and/or fainter than our sensitivity limit.

\section{Discussion and summary}

High spatial resolution mid-infrared observations of the three protostellar aggregates SSV 63, L1551 NE, and L1551-IRS5 have allowed us to unambiguously discover and verify two additional point-sources in the SSV 63 system. Also in the case of L1551 NE we could verify a very close companion, and 
conclude that the system is in an early evolutionary state. We could not resolve putative companions in L1551-IRS5 due to insufficient resolution, but see indications of extended MIR emission in this system. Our observations demonstrate the power of high spatial resolution mid-IR photometry to constrain the SED, and therefore the evolutionary status, of Class I sources only known from previous near-IR or radio continuum observations.

SSV 63 is of particular interest. Based on the geometrical appearance of multiple, bended, collimated outflows together with weak hints from NIR images, Terquem et al. (1999) postulated the existence of multiple protostars in that region. We have now unambiguously confirmed two of them. SSV 63 is located in an extremely complex environment, and our MIR observations are of great value to clarify the source geometries. It is unclear, however, if all five sources form a physically bound, multiple, protostellar system, though. While the two inner binaries might do so, it appears dubious that components with distances of more than $2000 \mathrm{AU}$ form gravitationally bound hierarchies. We speculate that we witness the arrangement of sources after hierarchical fragmentation of cloud cores. Filamentary dense cores break up into collapsing fragments having a scale size of several $1000 \mathrm{AU}$ (see, e.g., Motte et al. 2001, for the Orion B protoclusters NGC2 068 and NGC 2071 in Orion B), while physically bound protostellar systems might then appear and dynamically evolve on a scale of a few $100 \mathrm{AU}$ after the fragments have gone through isothermal gravitational collapse and stellar fragmentation (Sterzik et al. 2003). It will be interesting to extract dynamical information about these sources by determining their relative velocity dispersions through high resolution infrared spectroscopy (see Covey et al. 2006) and/or (sub)mm observations.

Acknowledgements. N.H. thanks A. Smette, E. Pantin, and H. Bouy. Support from the Fundação para a Ciência e a Tecnologia (Portugal) to N.C.S. in the form of a fellowship (reference SFRH/BPD/8116/2002) and a grant (reference POCI/CTE-AST/56453/2004) is gratefully acknowledged.

\section{References}

Bieging, J. H., \& Cohen, M. 1985, ApJ, 289, L5

Bontemps, S., Andre, P., \& Ward-Thompson, D. 1995, A\&A, 297, 98

Covey, K. R., Greene, T. P., Doppmann, G. W., \& Lada, C. J. 2006, AJ, 131, 512

Davis, C. J., Ray, T. P., Eisloeffel, J., \& Corcoran, D. 1997, A\&A, 324, 263

Duchêne, G., Bouvier, J., Bontemps, S., André, P., \& Motte, F. 2004, A\&A, 427, 651

Duchêne, G., Delgado-Donate, E., Haisch, Jr., K., Loinard, L., \& Rodriguez, L. 2006, ArXiv Astrophysics e-prints

Duquennoy, A., \& Mayor, M. 1991, A\&A, 248, 485

Kenyon, S. J., \& Hartmann, L. 1995, ApJS, 101, 117

Lada, C. J. 1987, in Star Forming Regions, ed. M. Peimbert, \& J. Jugaku, IAU Symp., 115, 1

Lagage, P. O., Pel, J. W., Authier, M., et al. 2004, The Messenger, 117, 12

Lim, J., \& Takakuwa, S. 2005, J. Korean Astron. Soc., 38, 237

Looney, L. W., Mundy, L. G., \& Welch, W. J. 2000, ApJ, 529, 477

Mathieu, R. D., \& Zinnecker, H. 2000, PASP, 112, 1512

Moneti, A., \& Reipurth, B. 1995, A\&A, 301, 721

Moriarty-Schieven, G. H., Powers, J. A., Butner, H. M., Wannier, P. G., \& Keene, J. 2000, ApJ, 533, L143

Motte, F., André, P., Ward-Thompson, D., \& Bontemps, S. 2001, A\&A, 372, L41 Patience, J. \& Duchêne, G. 2001, in ed. H. Zinnecker, \& R. Mathieu, IAU Symp., 181

Reipurth, B. 2000, AJ, 120, 3177

Reipurth, B., Rodríguez, L. F., Anglada, G., \& Bally, J. 2002, AJ, 124, 1045

Reipurth, B., Rodríguez, L. F., Anglada, G., \& Bally, J. 2004, AJ, 127, 1736

Rodríguez, L. F., Anglada, G., \& Raga, A. 1995, ApJ, 454, L149

Rodríguez, L. F., Porras, A., Claussen, M. J., et al. 2003, ApJ, 586, L137

Sterzik, M. F., Durisen, R. H., \& Zinnecker, H. 2003, A\&A, 411, 91

Strom, K. M., Strom, S. E., \& Vrba, F. J. 1976, AJ, 81, 308

Terquem, C., Eislöffel, J., Papaloizou, J. C. B., \& Nelson, R. P. 1999, ApJ, 512, L131

Zealey, W. J., Williams, P. M., Sandell, G., Taylor, K. N. R., \& Ray, T. P. 1992, A\&A, 262, 570 